\title{
An optical method for measuring exhaust gas pressure from an internal combustion engine at high speed
}

Felix C. P. Leach, Martin H. Davy, Dmitrij Siskin, Ralf Pechstedt, and David Richardson

Citation: Review of Scientific Instruments 88, 125004 (2017); doi: 10.1063/1.5005161

View online: https://doi.org/10.1063/1.5005161

View Table of Contents: http://aip.scitation.org/toc/rsi/88/12

Published by the American Institute of Physics

\section{Articles you may be interested in}

Prospects for magnetic field communications and location using quantum sensors

Review of Scientific Instruments 88, 125005 (2017); 10.1063/1.5003821

Picosecond-precision multichannel autonomous time and frequency counter

Review of Scientific Instruments 88, 125101 (2017); 10.1063/1.4997244

Optically transparent solid electrodes for precision Penning traps

Review of Scientific Instruments 88, 123101 (2017); 10.1063/1.5002180

An isocenter estimation tool for proton gantry alignment

Review of Scientific Instruments 88, 125102 (2017); 10.1063/1.4999287

Pulse stretcher with two beamsplitting elements for excimer laser pulses

Review of Scientific Instruments 88, 123113 (2017); 10.1063/1.5005123

Kriging modeling and SPSA adjusting PID with KPWF compensator control of IPMC gripper for mm-sized objects

Review of Scientific Instruments 88, 125003 (2017); 10.1063/1.4993663

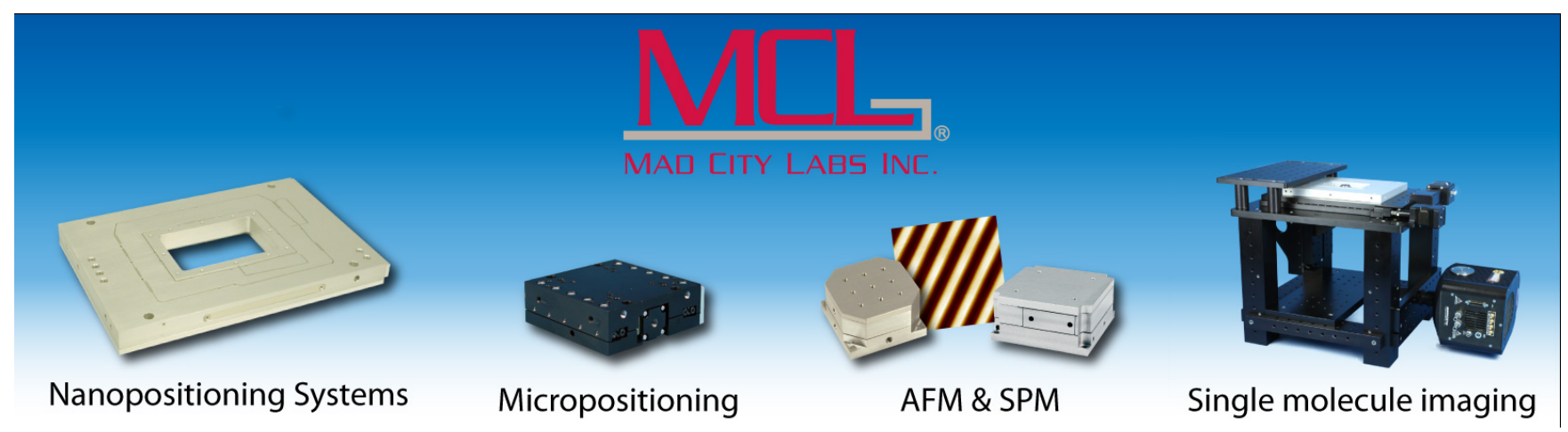




\title{
An optical method for measuring exhaust gas pressure from an internal combustion engine at high speed
}

\author{
Felix C. P. Leach, ${ }^{1, a)}$ Martin H. Davy, ${ }^{1}$ Dmitrij Siskin, ${ }^{2}$ Ralf Pechstedt, ${ }^{2}$ and David Richardson ${ }^{3}$ \\ ${ }^{1}$ Department of Engineering Science, University of Oxford, Oxford OX1 3PJ, United Kingdom \\ ${ }^{2}$ Oxsensis Ltd., Didcot OX11 OSG, United Kingdom \\ ${ }^{3}$ Powertrain Research, Jaguar Land Rover, Coventry CV4 7HS, United Kingdom
}

(Received 18 September 2017; accepted 20 November 2017; published online 11 December 2017)

\begin{abstract}
Measurement of exhaust gas pressure at high speed in an engine is important for engine efficiency, computational fluid dynamics analysis, and turbocharger matching. Currently used piezoresistive sensors are bulky, require cooling, and have limited lifetimes. A new sensor system uses an interferometric technique to measure pressure by measuring the size of an optical cavity, which varies with pressure due to movement of a diaphragm. This pressure measurement system has been used in gas turbine engines where the temperatures and pressures have no significant transients but has never been applied to an internal combustion engine before, an environment where both temperature and pressure can change rapidly. This sensor has been compared with a piezoresistive sensor representing the current state-of-the-art at three engine operating points corresponding to both light load and full load. The results show that the new sensor can match the measurements from the piezoresistive sensor except when there are fast temperature swings, so the latter part of the pressure during exhaust blowdown is only tracked with an offset. A modified sensor designed to compensate for these temperature effects is also tested. The new sensor has shown significant potential as a compact, durable sensor, which does not require external cooling. Published by AIP Publishing. https://doi.org/10.1063/1.5005161
\end{abstract}

\section{INTRODUCTION}

Internal combustion engine exhaust is typically a harsh environment, a pulsating flow experiencing temperatures 0 $1000^{\circ} \mathrm{C}$ and pressures of ambient- 5 barG and a rapid range of transients between those extremes. Measurement of these conditions is vital for successful engine design, the conditions in the exhaust effect engine efficiency, emissions, and aftertreatment efficacy. Tuning of the exhaust (using the geometry of the exhaust manifold to ensure exhaust pulses from different cylinders act constructively) can increase engine efficiency by aiding the gas exchange process (on the other hand poorly tuned exhausts will have a negative impact on efficiency). ${ }^{1-4}$ Exhaust manifold tuning also has a significant impact on the engine noise. On an engine fitted with a turbocharger, measuring and understanding this flow is essential for proper turbocharger matching-again having significant impacts on engine efficiency. ${ }^{5}$ Turbochargers are normally fitted as close to the exhaust ports as possible to minimize energy losses, and so access for measurement can pose a challenge.

Crank angle resolved exhaust pressure from experiments has become an important parameter in both CFD analysis of engines and 1D models such as GT-Power as part of socalled three-pressure analysis (TPA) using inlet, cylinder, and exhaust pressures. ${ }^{6}$ This helps us to define gas exchange quantities and impacts important engine parameters such as residual gas fraction, scavenging, and fuel short circuiting. In addition these models are essential in designing port flow and valve lift requirements.

\footnotetext{
a) Author to whom correspondence should be addressed: felix.leach@eng. ox.ac.uk
}

The current state-of-the-art in exhaust pressure measurement is generally accepted to be piezoresistive absolute pressure sensors. Such sensors operate on a resistive principle where the resistance of a semiconductor changes substantially with mechanical deformation. ${ }^{7}$ Typically the pressure is applied to a thin steel diaphragm and transferred through silicone oil to a silicon semiconductor. ${ }^{8}$ Such sensors typically operate at temperatures of up to $140{ }^{\circ} \mathrm{C}$ and $10 \mathrm{barG}$ (without cooling). Given typical exhaust temperatures, sensors are fitted with radiation shields and either need to be mounted some distance from the exhaust (losing dynamic response) or need to be cooled; the latter introduces expense and bulk (due to the comparative simplicity of the high temperature optical sensing system, considerable cost savings might be made). Given the harsh conditions in the exhaust, piezoresistive transducers are not typically constantly exposed to the exhaust gases, normally being fitted inside an adaptor designed only to expose the sensor on the application of some control signal. Even with cooling and non-constant exposure sensor durability is an issue, with exhaust sensor durability significantly less good than similar sensors fitted in the inlet system of an engine. ${ }^{9}$ Fast response piezoresistive transducers are an important part of engine R\&D but are not typically fitted to production engines (and nor is there currently a need for them to be).

Optical pressure sensors, such as those tested in this work, evaluate pressure by measuring the size of an optical cavity, the size of which varies with applied pressure. ${ }^{10}$ A schematic of this type of sensor is shown in Fig. 1. The cavity size is determined using optical interferometry and evaluating the light signal using an interrogator to convert the light to an electrical output. Such a system has numerous advantages including 


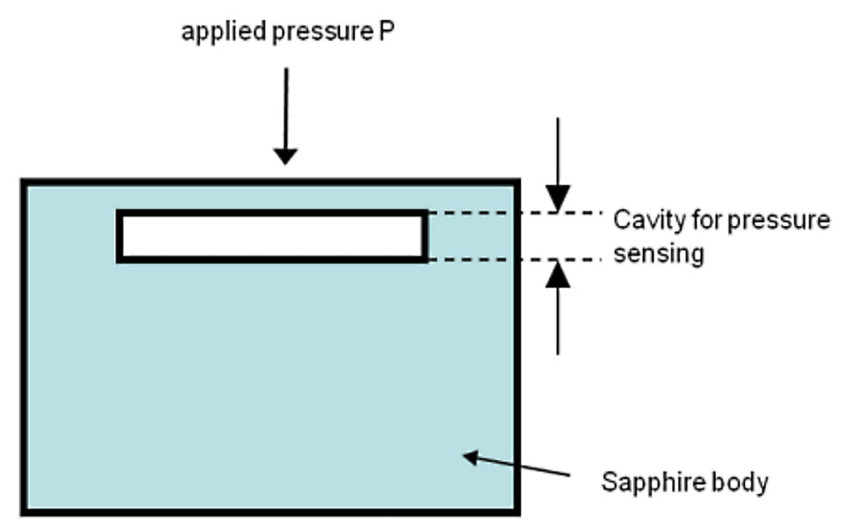

FIG. 1. Schematic of an optical pressure sensing element showing the sapphire body and the cavity (the size of which is measured to determine pressure).

a high robustness to electrical noise (as the signal is a light signal), long lifetimes, and high sensitivity. Previous deployments of this type of sensor on a combined cycle gas turbine (CCGT) power station have demonstrated lifetimes in excess of 16500 h. $^{11}$

The aim of this work is to compare this new sensor with the established state-of-the-art at a variety of engine exhaust conditions representative of what might be found on modern vehicles.

\section{EXPERIMENTAL METHODOLOGY}

In order to observe the capabilities of the new sensor, the optical sensor was mounted alongside a comparison sensor in the exhaust manifold of a diesel single cylinder research engine at the University of Oxford. The engine is a Ricardo Hydra base, fitted with a piston, cylinder head, and fuel injection equipment replicating the Jaguar Land Rover AJ200D "Ingenium" engine, and as such can be seen as a single cylinder version of that engine. The single cylinder engine and test cell instrumentation have been comprehensively described in Refs. 12 and 13. Because the engine is not equipped with a turbocharger, exhaust pressure is provided by means of a back pressure valve and inlet air pressure via an external compressor. The exhaust valve opens at 127 crank angle degrees (CAD) and closes at $-337 \mathrm{CAD}$ ( $0 \mathrm{CAD}$ corresponds to top dead centre (TDC) firing).

Two optical sensors were tested, one "standard" and one modified to take into account the high temperature transients experienced in engine exhaust. The general appearance of the optical sensors tested is shown in Fig. 2. Some small

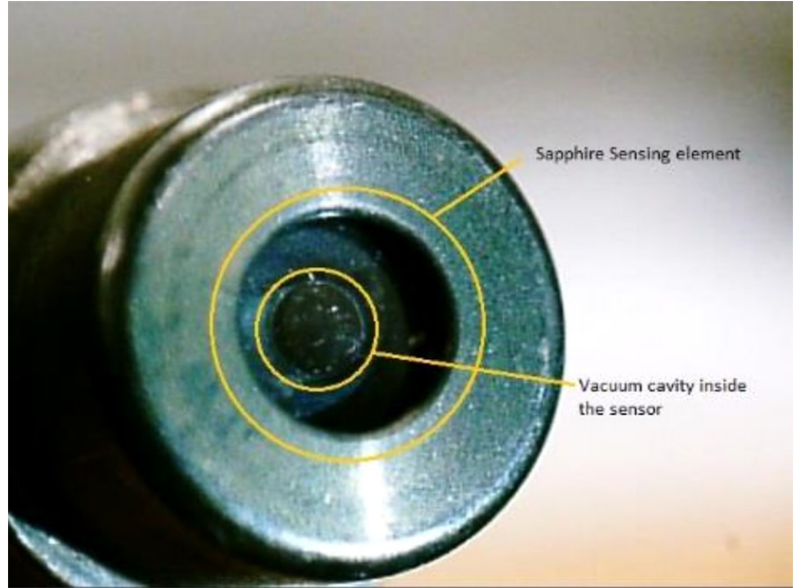

FIG. 3. Standard optical sensor, showing the sensing element and the vacuum cavity.

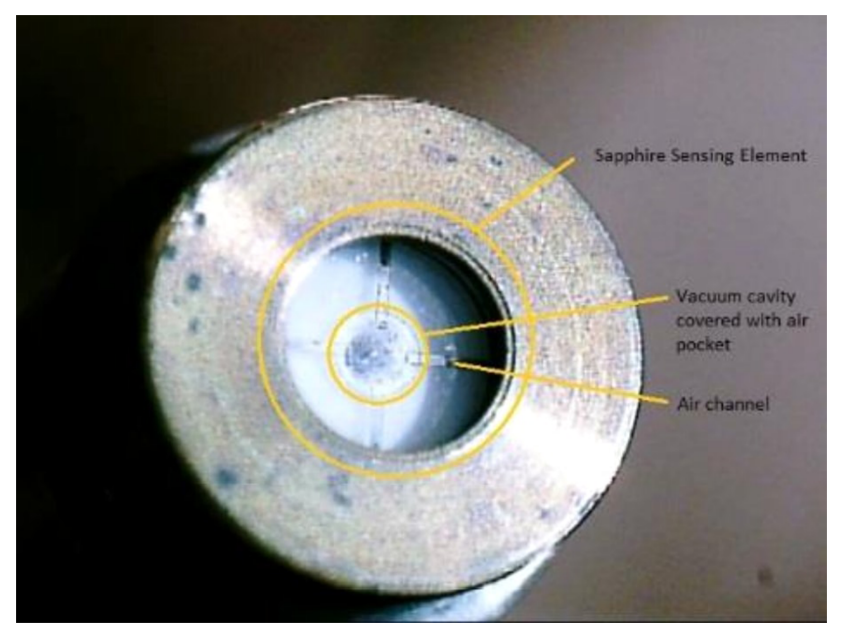

FIG. 4. Modified optical sensor, showing the layer of sapphire with air channels which has been layered over the top of the standard optical sensor (Fig. 3).

modifications were applied to one sensor in order to attempt to compensate for the fast temperature transitions that were expected at the sensing element front (located in the sensor head).

The standard optical sensor is Oxsensis PT1100 $\mathrm{e}^{10}$ which is shown in Fig. 3. The standard optical sensor has a vacuum cavity formed of the sensor sensing element base and the diaphragm as in Fig. 1. The modified optical sensor is again Oxsensis PT1100e using the same sensing element structure; however, the diaphragm is covered with a layer of sapphire

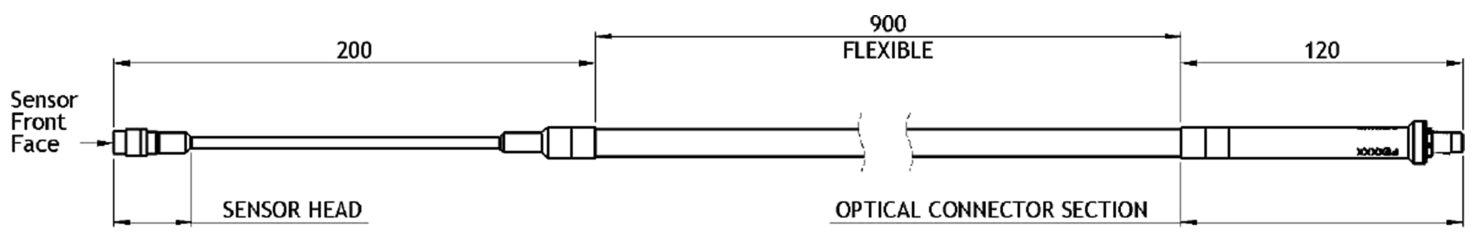




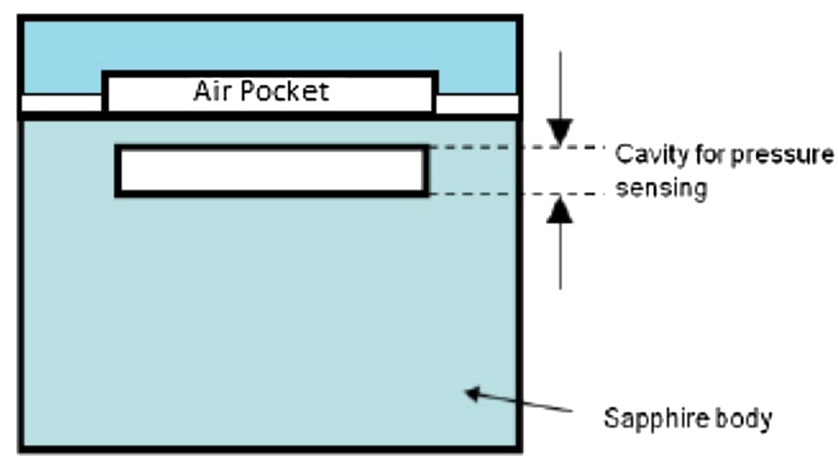

FIG. 5. Schematic diagram of modified Oxsensis sensing element; note the sapphire layer which has been applied over the top of the diaphragm.

processed to allow air through 4 channels on the sides of the sensor in order to have an air pocket over the diaphragm in an attempt to reduce the temperature transients experienced by the sensor. An image of the modified sensor head is shown in Fig. 4, and a schematic of these modifications is shown in Fig. 5.

The optical sensor is an absolute pressure transducer; however, its reading needs to be temperature compensated to give static absolute pressure. The signal interrogator used in this test does not support spectral measurement of sensor element temperature and so dynamic absolute pressure is only currently able to be output. If a different type of an interrogator is used, the sensor is capable of measuring static pressure, dynamic pressure, and temperature. In this case, the engine is fitted with a GE Druck UNIK5000 series pressure transducer, capable of measuring 0-5 barG-this is mounted around $5 \mathrm{~m}$ after the tapping for it to eliminate dynamic effects and high

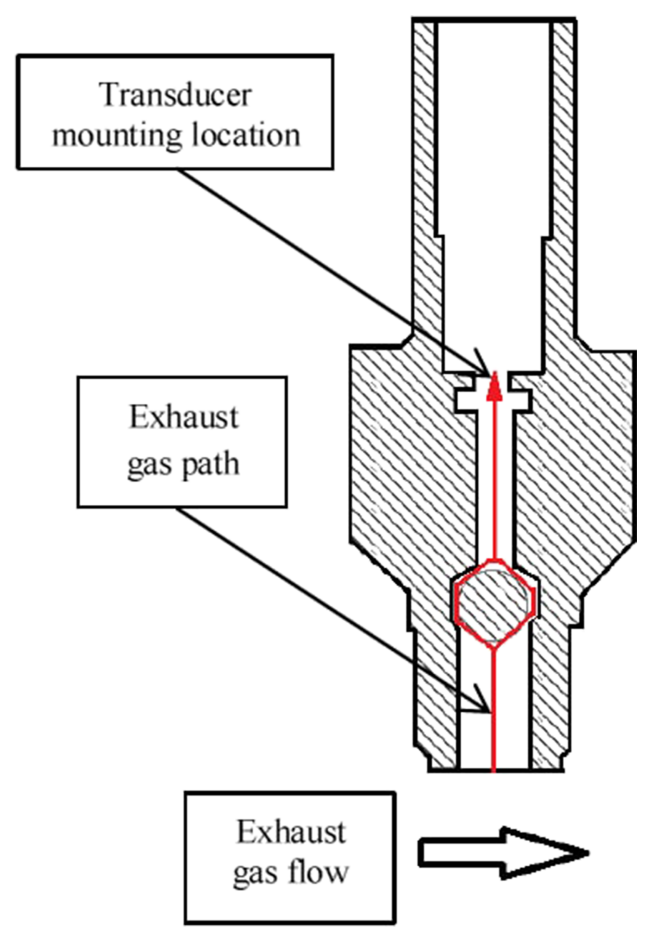

FIG. 6. Cooled switching adaptor in which the reference transducer is mounted. The exhaust gas path is shown in red. Adapted from Ref. 15.

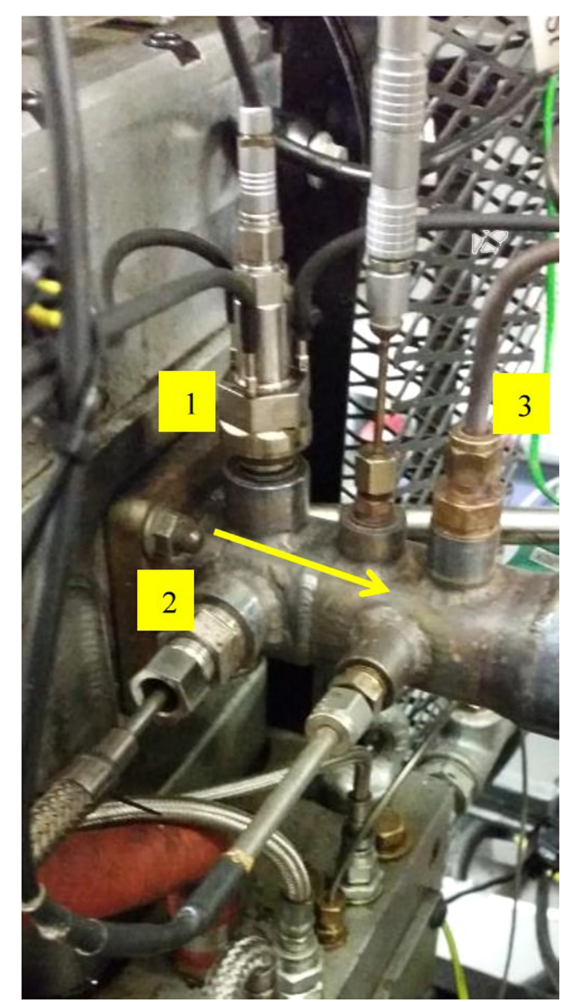

FIG. 7. The reference transducer and optical sensor installed on the engine; the smaller size of the optical sensor is notable. (1) Reference transducer, (2) optical sensor, (3) "slow" pressure measurement. The arrow indicates the direction of exhaust flow.

temperatures. This sensor is used to set the exhaust back pressure and is referred to as the "slow" pressure sensor. This "slow" pressure is used to give an absolute magnitude (effectively a DC offset) to the optical sensor data. The results from both the optical sensor and the comparison sensor are presented as absolute pressure in bar in Figs. 9-14.

The comparison sensor (referred to as the standard transducer throughout) used in this work is a Kistler 4075A10V200S piezoresistive pressure sensor mounted in a Kistler 7533B12 cooled switching adaptor. ${ }^{14}$ This transducer can measure $0-10$ bars absolute pressure in a temperature range of $0-120{ }^{\circ} \mathrm{C}$, and the cooling water was fixed at a temperature of $30{ }^{\circ} \mathrm{C}$. The signal from this transducer was amplified by a Kistler 4665 amplifier before being logged. A cut-away of the cooled switching adaptor is shown in Fig. 6. This shows that in order to protect the transducer, there is quite a long, convoluted gas path between the exhaust gas flow and the transducer itself.

Both exhaust pressure sensors were mounted as close as possible to the exhaust port, approximately $2 \mathrm{~cm}$ downstream

TABLE I. Engine operating points

\begin{tabular}{|c|c|c|c|}
\hline Test point ID & 1 & 2 & 3 \\
\hline Engine speed (rpm) & \multicolumn{2}{|c|}{1500} & 2000 \\
\hline Exhaust temperature $\left({ }^{\circ} \mathrm{C}\right)$ & 320 & 450 & 750 \\
\hline Exhaust backpressure (barG) & 0.45 & 0.9 & 1.75 \\
\hline
\end{tabular}




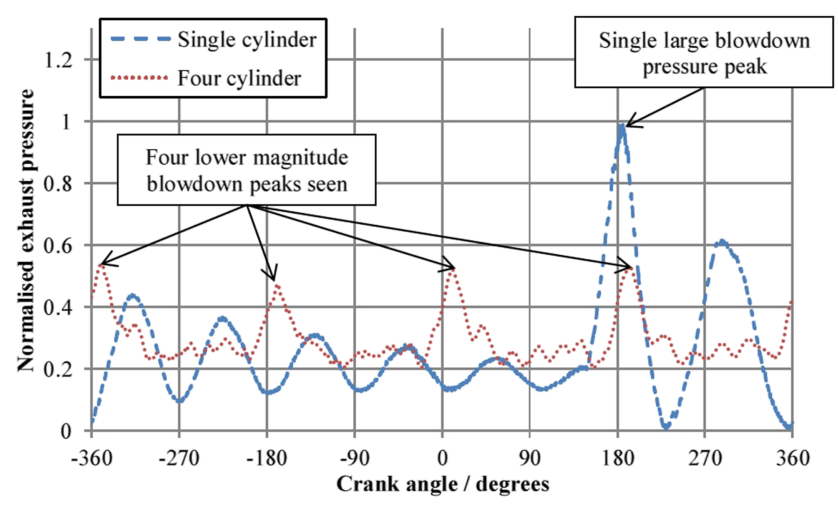

FIG. 8. Comparison of exhaust pressures between a single- and four-cylinder engine at the same operating point. Lower blowdown peaks and suppressed resonances can be seen in the four-cylinder engine due to cylinder-cylinder interactions. $0^{\circ}$ on the ordinate corresponds to TDC firing.

of the start of the exhaust manifold at the same point in the manifold, orthogonal one to another as shown in Fig. 7. Both the standard transducer and the optical sensor were logged simultaneously by an AVL advanced indiset 642 at a 1 CAD resolution (which corresponds to $9 \mathrm{kHz}$ at an engine speed of $1500 \mathrm{rpm}$ and $12 \mathrm{kHz}$ at $2000 \mathrm{rpm}$ ). Other parameters (including the "slow" pressure data) were logged by the Sierra-CP Cadet software at $1 \mathrm{~Hz}$.

The engine was run at a variety of test conditions in order to explore the operation of the transducer under different conditions. The details of the engine operating points are shown in Table I. Test points 1 and 2 are typical light load operating points, and test point 3 representative of full load running.

It should be noted that exhaust pressures observed in a single cylinder engine are not the same as would be observed in a typical multi-cylinder engine. Figure 8 illustrates this effect, comparing exhaust pressures from a single- and fourcylinder engine, both running at the same operating point. A large single blowdown pulse is seen from the single-cylinder engine, corresponding to the point where the exhaust valve opens. The four-cylinder engine gives four blowdown pulses (as would be expected) but at a much lower overall magnitude compared to the single cylinder engine due to cylindercylinder interactions. The corresponding resonances on the
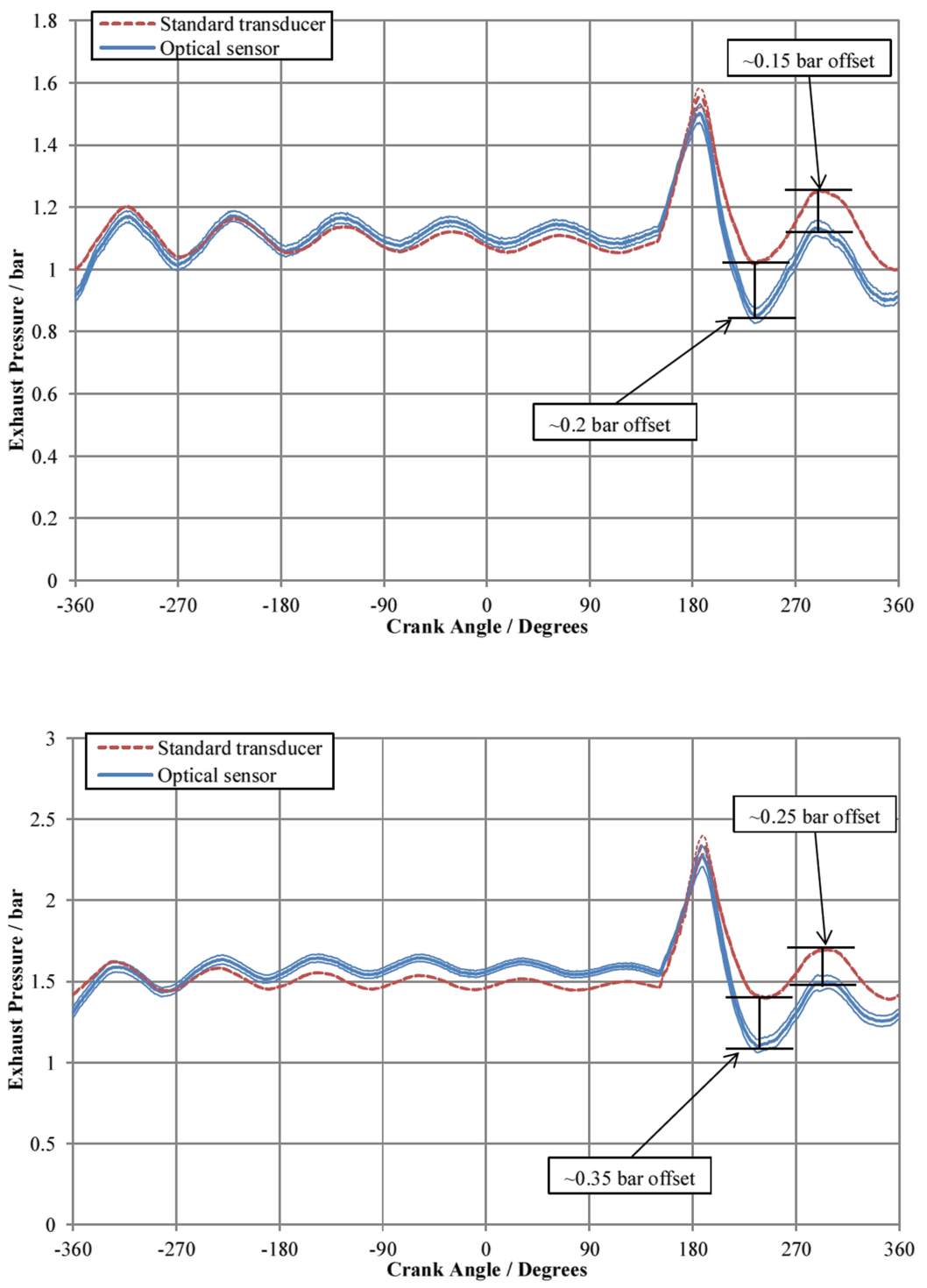

FIG. 9. Comparison between the optical sensor and the standard transducer at test point 1 . A very good correlation between the two sensors is observed during initial blowdown, but the optical sensor reads lower than the standard transducer subsequently. $0^{\circ}$ on the ordinate corresponds to TDC firing. The error bands correspond to $\pm \sigma$.
FIG. 10. Comparison between the optical sensor and the standard transducer at test point 2 . The same trends as seen at test point 1 are observed, albeit the offset is increased. $0^{\circ}$ on the ordinate corresponds to TDC firing. The error bands correspond to $\pm \sigma$. 

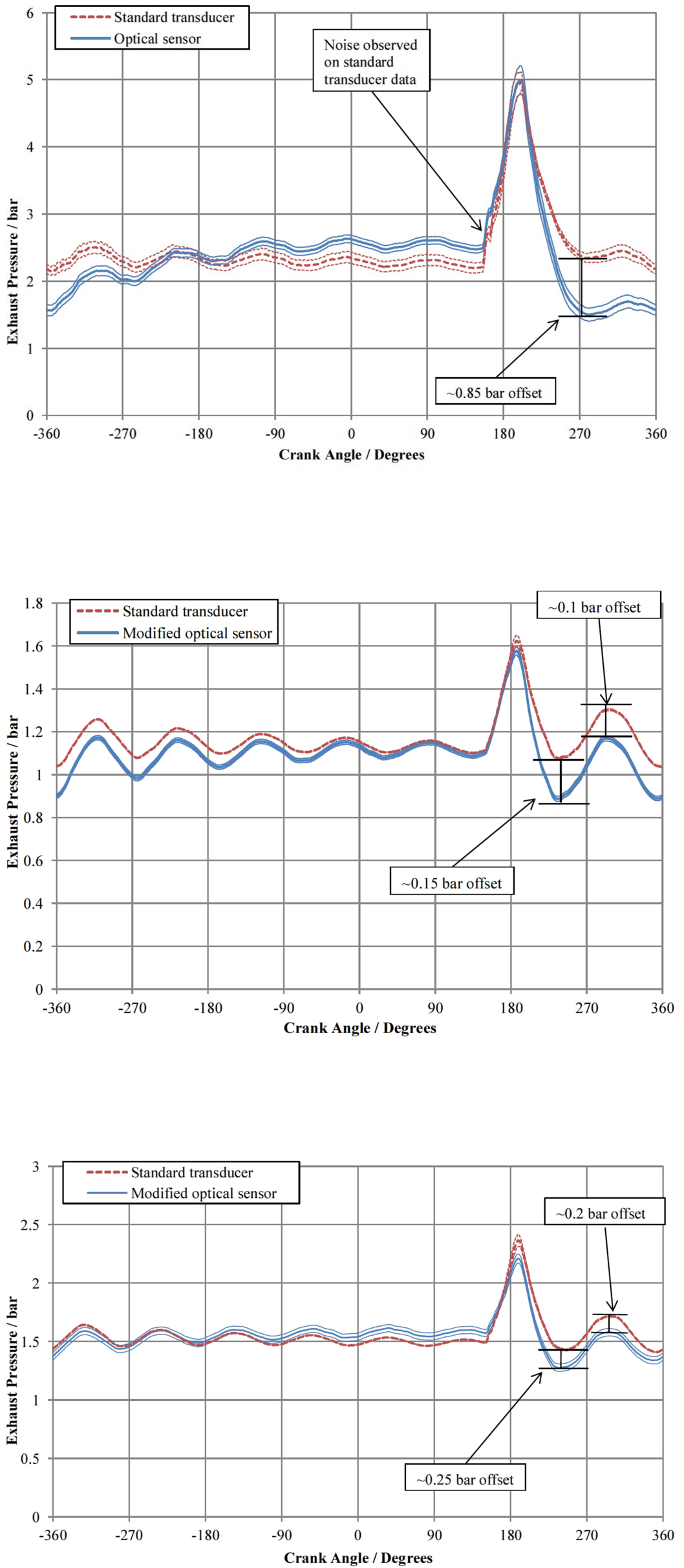

FIG. 11. Comparison between the optical sensor and the standard transducer at test point 3 . Similar trends to the other operating points are noted; however, there is significant noise visible on the standard transducer data during initial blowdown not seen on the optical sensor. $0^{\circ}$ on the ordinate corresponds to TDC firing. The error bands correspond to $\pm \sigma$.
FIG. 12. Comparison between the modified optical sensor and the standard transducer at test point 1 . A very good correlation between the two sensors is observed during initial blowdown, but the optical sensor reads lower than the standard transducer subsequently, however, with a smaller offset compared to the unmodified optical sensor (Fig. 9). $0^{\circ}$ on the ordinate corresponds to TDC firing. The error bands correspond to $\pm \sigma$.
FIG. 13. Comparison between the modified optical sensor and the standard transducer at test point 2. Again the modified optical sensor gives a smaller offset compared to the unmodified optical sensor (Fig. 10). $0^{\circ}$ on the ordinate corresponds to TDC firing. The error bands correspond to $\pm \sigma$. 


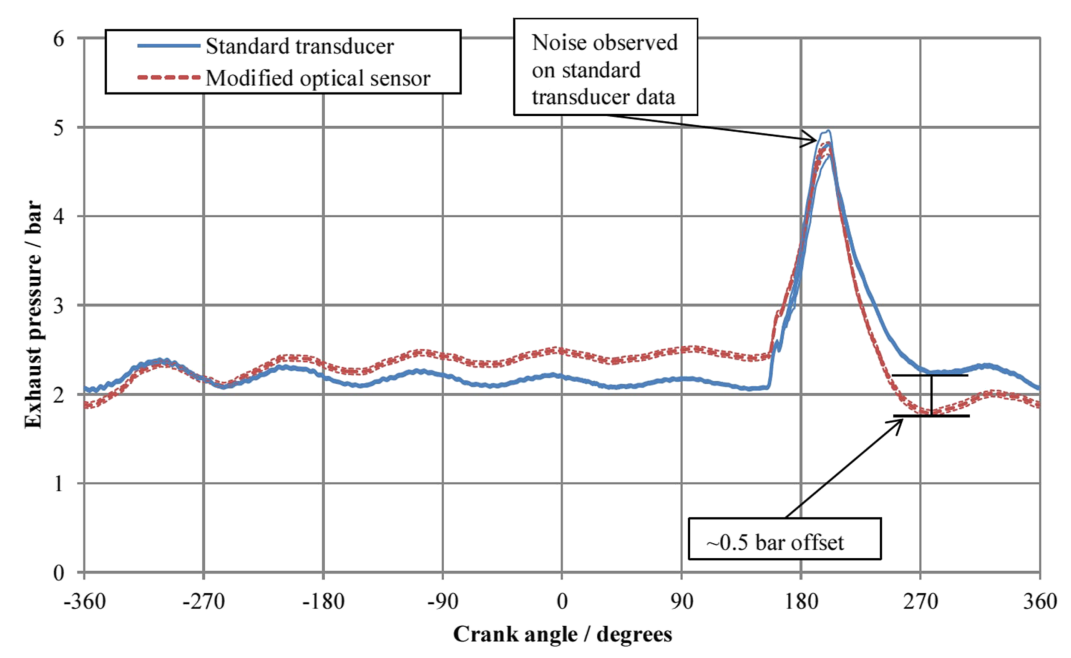

FIG. 14. Comparison between the modified optical sensor and the standard transducer at test point 3 . The modified optical sensor is shown to perform better than the unmodified (Fig. 11), albeit still with significant offset. The noise on the standard transducer during blowdown is particularly visible here. $0^{\circ}$ on the ordinate corresponds to TDC firing. The error bands correspond to $\pm \sigma$. four-cylinder engine are much lower as well, for the same reason.

\section{RESULTS AND DISCUSSION}

\section{A. Optical sensor}

Figure 8 shows the comparison between the optical sensor and the standard transducer at test point $1(0.45 \mathrm{barG}$, $320^{\circ} \mathrm{C}$ ). A very good agreement between the two sensors is observed from -180 to $200 \mathrm{CAD}$; however, during the second part of blowdown, there is a clear deviation between the two sensors; however, both still capture the same general trend. The optical sensor is underreading with a maximum underread of around 0.2 bar. This is thought to be due to the temperature transient at the front of the sensor, resulting in temperature expansion of the sensing element. As the exhaust valve opens there will be an initial large temperature transient (say $120^{\circ} \mathrm{C}$ at this test point), and this will cause the optical probe to underread because of the thermal expansion of the cavity. Very simplified analysis of pressure error caused by temperature can be made assuming purely axial expansion of the sapphire ring around the vacuum cavity. Using this analysis, a temperature spike of $50{ }^{\circ} \mathrm{C}$ would theoretically cause a 0.15 bar error, which is close to the observed errors. The same simplified analysis shows this would result in a 0.36 bar pressure reading error with a $120^{\circ} \mathrm{C}$ temperature transientthis suggests that the transient experienced by the sapphire is approximately $70^{\circ} \mathrm{C}$. As the temperature transient dissipates, this offset is reduced, and the signals are back "line-on-line" by -180 CAD.

Figure 10 shows the comparison between the optical sensor and the standard transducer at test point $2(0.9$ barG, $450{ }^{\circ} \mathrm{C}$ ). Again the trends visible at test point 1 are observed here; however, the magnitude of the offset between the two sensors is increased, most likely due to the higher temperatures at this test point.

Figure 11 shows the comparison between the optical sensor and the standard transducer at test point 3 (1.75 barG, $750{ }^{\circ} \mathrm{C}$ ). At these highest temperatures, it is clear that the offset introduced into the optical sensor is large-around
0.85 bar; however, it still matches the general trend, and the blowdown event is well captured. Also of note is the noise that is clearly visible in the standard transducer during the blowdown event, much higher than the noise; this effect is also visible at test points 1 and 2 but is less marked at these lower pressure test points. It is likely that this is being caused by a Helmholtz resonance due to the cooled switching adaptor in which the standard transducer is mounted (Fig. 6). This is because the exhaust gas flow is passing orthogonal to the adaptor, which has a series of small cavities between the main exhaust flow and the transducer. This is leading to "ringing" which is clearly visible in the standard transducer signal during the blowdown pulse (indeed analysis of individual cycles shows the high frequency "ringing" type behavior in the signal).

\section{B. Modified optical sensor}

The modified probe was tested alongside the same standard transducer at the same engine operating points, although inevitably it was not possible to ensure the engine was running at precisely the same exhaust temperature and pressure, so the modified and unmodified optical sensor data cannot be compared back to back.

Figure 12 shows the comparison between the optical sensor and the standard transducer at test point 1 ( $0.45 \mathrm{barG}$, $320^{\circ} \mathrm{C}$ ). The trends observed with the unmodified optical sensor (Fig. 9) are still clearly visible; however, the magnitude of the offset has been reduced, showing that the modifications have had some benefit, however, not fully resolved the difference.

Figure 13 shows the comparison between the optical sensor and the standard transducer at test point $2(0.9 \mathrm{barG}$, $450{ }^{\circ} \mathrm{C}$ ). Again the modified sensor is giving a smaller offset than the unmodified sensor (around a 30\% improvement).

Figure 14 shows the comparison between the optical sensor and the standard transducer at test point 3 (1.75 barG, $750{ }^{\circ} \mathrm{C}$ ). At these highest temperatures, the modified optical sensor still has difficulty matching the second half of the blowdown; however, again there is a significant improvement with the modified sensor compared to the unmodified optical sensor. 
Being an optical sensor, it might be expected that deposits from engine exhaust would, over time, degrade the performance of the sensor. At prolonged lower temperature running with the modified optical sensor we observed some of thiswith deposits clogging the channels seen in Fig. 4, however, with high temperature running these were all removed. No deposits were observed on the standard optical sensor. When deposits were observed with the modified optical sensor, their effect was to degrade the frequency response of the sensoreffectively slightly modifying the pressure response of the sensor. It is thought that this is a mechanical effect (blocking the channels changes the response of the size of the cavity to pressure) rather than an optical one; previous tests with the standard sensor have shown no change in response with contamination.

\section{CONCLUSIONS}

A new optical pressure sensor has been used to measure exhaust pressure from a single cylinder diesel engine at three operating points. From the data, the following conclusions are drawn:

- The optical sensor has been able to match the trends of engine exhaust pressure when compared to a current state-of-the-art piezoresistive sensor; however, the impact of high temperature swings causes some significant offsets between the two sensors.

- The optical sensor's sensitivity to temperature is because of the thermal expansion of its sensing element. A modified optical sensor had some effect in reducing this; however, a significant offset remained.

- Helmholtz ringing was observed on the standard transducer during the exhaust blowdown period, which was not observed with the optical sensor because of its direct mounting.

Future developments to address the temperature sensitivity of the optical sensor will include different shielding of the sensor element if practical and/or using multiple optical cavities to enable fast temperature compensation of the sensing element. The latter would require a new signal interrogator that utilises both dual wavelength real time dynamic pressure monitoring and fast spectral resolution of multiple cavities.

Overall the optical sensor has shown promise, and for applications with limited access, its small size and no need for external cooling are significant advantages. The optical sensor has advantages over the existing type of transducers due to its ability to be mounted directly in the exhaust (and thereby avoiding Helmholtz ringing effects). In addition its expected durability (as demonstrated in previous applications in harsh environments) is a benefit compared to piezoresistive sensors, which are challenged in this area.

\section{ACKNOWLEDGMENTS}

The authors would like to thank Jaguar Land Rover Limited and the University of Oxford John Fell fund for financial support. The authors would also like to thank BP International for supplying the diesel fuel. The authors would like to thank Liyah Dholiwar for undertaking much of the engine testing and the Department of Engineering Science technicians and maintenance teams for facilities support. The authors would like to thank Alice Martin for data analysis assistance with the pressure signals.

\section{NOMENCLATURE}

barA bar absolute

barG bar gauge

CAD crank angle degrees

CCGT combined cycle gas turbine

CFD computational fluid dynamics

TDC top dead centre

TPA three-pressure analysis

${ }^{1} \mathrm{R}$. Stone, Introduction to Internal Combustion Engines, 4th ed. (Palgrave Macmillan, 2012).

${ }^{2}$ G. Sammut and A. C. Alkidas, "Relative contributions of intake and exhaust tuning on SI engine breathing-A computational study," SAE Technical Paper 2007-01-0492, SAE International, 2007.

${ }^{3}$ G. P. Blair, D. O. Mackey, M. C. Ashe, and G. F. Chatfield, "Exhaust tuning on a four-stroke engine, experimentation and simulation," SAE Technical Paper 2001-01-1797, SAE International, 2001.

${ }^{4}$ C. E. Möller, P. Johansson, B. Grandin, and F. Lindström, "Divided exhaust period - a gas exchange system for turbocharged SI engines," SAE Technical Paper 2005-01-1150, SAE International, 2005.

${ }^{5} \mathrm{~A}$. Lefebvre and S. Guilain, "Modelling and measurement of the transient response of a turbocharged SI engine," SAE Technical Paper 2005-01-0691, SAE International, 2005.

${ }^{6} \mathrm{~K}$. Goerg and T. Morel, "Use of TPA (Three-Pressure analysis) to obtain burn rates and trapped residuals," in Proceedings of GT-SUITE Conference, 2005.

${ }^{7}$ Demystifying Piezoresistive Pressure Sensors, Maxim integrated, application note 871, (2002).

${ }^{8}$ Kistler data sheet 000-003.

${ }^{9}$ Kistler data sheet 000-064.

${ }^{10}$ PT1100e Dynamic Pressure Sensor datasheet v1.4.

${ }^{11}$ A. Winterburn, R. Pechstedt, F. Maillaud, T. Rödiger, O. Lammel, and P. Griebel, Extension of an optical dynamic pressure sensor to measure temperature and absolute pressure in combustion applications," in Proceedings of 6th International Gas Turbine Conference, Brussels, 2012.

${ }^{12} \mathrm{~F}$. Leach, M. Davy, A. Weall, and B. Cooper, "Comparing the effect of a swirl flap and asymmetric inlet valve opening on a light duty diesel engine," SAE Technical Paper 2017-01-2429, SAE International, 2017.

${ }^{13}$ F. Leach, R. Ismail, M. Davy, A. Weall, and B. Cooper, "Comparing the effect of fuel/air interactions in a modern high-speed light-duty diesel engine," SAE Technical Paper 2017-24-0075, SAE International, 2017.

${ }^{14}$ Kistler data sheet 002-614.

${ }^{15}$ J. C. Beale and R. D. Reitz, "Modeling spray atomization with the Kelvin-Helmholtz/Rayleigh-Taylor hybrid model," Atomization Sprays 9, 623-650 (1999) 